\title{
Parameter Estimation of an Epidemic Model with State Constraints
}

\author{
Gabriela Marinoschi ${ }^{1}$
}

Accepted: 5 August 2021 / Published online: 8 September 2021

(c) The Author(s), under exclusive licence to Springer Science+Business Media, LLC, part of Springer Nature 2021

\begin{abstract}
We consider a mathematical model with five compartments relevant to depict the feature of a certain type of epidemic transmission. We aim to identify some system parameters by means of a minimization problem for a functional involving available measurements for observable compartments, which we treat by an optimal control technique with a state constraint imposed by realistic considerations. The proof of the maximum principle is done by passing to the limit in the conditions of optimality for an appropriate approximating problem. The proof of the estimates for the dual approximating system requires a more challenging treatment since the trajectories are not absolutely continuous, but only with bounded variation. These allow to pass to the limit to obtain the conditions of optimality for the primal problem and lead to a singular dual backward system with a generalized solution in the sense of measure. As far as we know, this approach developed here for the identification of parameters in an epidemic model considering a state constraint related to the actions undertaken for the disease containment was not addressed in the literature and represents a novel issue in this paper.
\end{abstract}

Keywords Inverse problems $\cdot$ Control with state constraints $\cdot$ Necessary conditions of optimality $\cdot$ Epidemics $\cdot$ SARS-CoV-2

Mathematics Subject Classification 49N45 - 49Jxx · 49K15 - 92D30 · 9310

\section{Introduction}

We study an identification problem in relation with a nonlinear system of five differential equations describing an epidemic evolution, focusing on the estimation of

$凶$ Gabriela Marinoschi

gabriela.marinoschi@acad.ro

1 "Gheorghe Mihoc-Caius Iacob" Institute of Mathematical Statistics and Applied Mathematics of the Romanian Academy, Calea 13 Septembrie 13, Bucharest, Romania 
some parameters of the system connected with the actions undertaken for the epidemic containment.

We consider the following nonlinear system of differential equations

$$
\begin{aligned}
& S^{\prime}(t)=-\beta_{I}(t) S(t) I(t)-\beta_{A}(t) S(t) A(t)+\xi R(t), \\
& A^{\prime}(t)=\beta_{I}(t) S(t) I(t)+\beta_{A}(t) S(t) A(t)-\left(\sigma+\mu_{A}+l_{A}(t)\right) A(t), \\
& I^{\prime}(t)=\sigma A(t)-\left(\mu_{I}+l_{I}(t)\right) I(t), \\
& L^{\prime}(t)=l_{A}(t) A(t)+l_{I}(t) I(t)-\mu_{L} L(t), \\
& R^{\prime}(t)=\mu_{A} A(t)+\mu_{I} I(t)+\mu_{L} L(t)-\xi R(t),
\end{aligned}
$$

for a.a. $t>0$, with the initial conditions

$$
S(0)=S_{0}, A(0)=A_{0}, I(0)=I_{0}, L(0)=L_{0}, R(0)=R_{0} .
$$

This system describes a mathematical model of an epidemic with five compartments, represented at time $t$ by the susceptible $S(t)$ (healthy individuals which can acquire the disease), infected asymptomatic $A(t)$ (individuals who have acquired the disease, have no symptoms or only mild ones and have not yet been confirmed), infected with symptoms but not confirmed yet $I(t)$, detected by medical tests and isolated (e.g., by hospitalization) $L(t)$, and recovered $R(t)$. In this model we assume that there are two classes of infected populations $A$ and $I$ which are both infectious, that is individuals from each these classes can spread the virus to the susceptible individuals with the rates $\beta_{I}(t)$ and $\beta_{A}(t)$, respectively. The number of individuals in these classes is not known since they are not still checked and confirmed. Since the identification of the parameters we propose here is considered to be performed on short intervals of time, we skip in this model the extinct (from any reason) and the newborns, because their number is negligible compared with the large number of people in the relevant compartments for a disease with large transmissibility and low mortality. Thus, in the perspective of the identification problem approached in this work, we assume that the model contains relevant classes of populations evolving during this epidemic. The study presented in this paper can be applied, of course, to a model completed with equations for other compartments of interest.

This model is suitable to describe the infection with SARS-CoV-2, since it is known that the asymptomatic individuals infected with this virus ( $A$ in this model) can transmit the disease (see [1]). The model we consider here may be seen as a reduced version of more general descriptions of a SARS-CoV-2 epidemic, see e.g., those presented and analyzed e.g., in [2-4] which include many compartments, in particular of hospitalized individuals exhibiting more or less serious symptoms. These classes are included in the compartment $L$ of our model.

The model is given here in a normalized form, that is the real number of individuals at time $t$ in each class is divided by the total population at time $t$, given by the sum of all components $N(t):=S(t)+A(t)+I(t)+L(t)+R(t)$. By (1.1)-(1.5) we note that

$$
\frac{d}{d t}(S(t)+A(t)+I(t)+L(t)+R(t))=0,
$$


so that we deduce that the total population $N(t)$ remains unchanged in time, that is

$$
S(t)+A(t)+I(t)+L(t)+R(t)=S_{0}+A_{0}+I_{0}+L_{0}+R_{0}=N, \text { for all } t \geq 0 .
$$

Since each term in these sums is nonnegative (as representing a fraction of population), then it is bounded by $N$. Moreover, since we work with fractions of population, $N$ is actually equal to 1 , but we shall keep it written as $N$ to precisely indicate where it occurs.

We are going to explain the meaning of the parameters in order to sustain the mathematical assumptions we shall set for them. The functions $\beta_{I}$ and $\beta_{A}$ represent the average number of individuals infected in unit time by an infective symptomatic and asymptomatic. The values $\sigma, l_{A}$ and $\mu_{A}$ are, respectively, the rates at which an asymptomatic can become symptomatic, is detected and isolated, and is recovered. Similarly, $l_{I}$ and $\mu_{I}$ are the rates at which a symptomatic is detected and recovered, respectively. The isolated individuals recover with the rate $\mu_{L}$. As in many other transmissible diseases, it was evidenced (see e.g., [5]) that in the case of infection with SARS-CoV-2 the immunity is gained for a short time only, so that a recovered individual can be infected again with the virus after some time and return into the susceptible compartment with the rate $\xi$. The removal rates $\mu_{I}, \mu_{A}, \mu_{L}$ and $\xi$ are constant, being represented by the inverse of the average healing time for a symptomatic, asymptomatic, infectious treated in the hospital and the inverse of the loss immunity time, respectively.

If one considers the compartment $L$ as containing all confirmed isolated individuals, the time dependent parameters $l_{A}$ and $l_{I}$ are the rates of detection, relative to asymptomatic and symptomatic cases, respectively (see e.g., $[3,6]$ ), that is the percentage of infections that are discovered. They may also reflect the number of tests performed over the population and can be modified by enforcing sustained actions, as for example a massive testing campaign (see [7]). The rate $l_{I}$ may be larger than $l_{A}$, because a symptomatic individual is more likely to be tested. Here, we assume that not all confirmed individuals remain isolated, so that $L$ contains only a part of the confirmed individuals and note that $L$ does not returns individuals in the infected classes in our model. Thus, we interpret the rates $l_{A}$ and $l_{I}$ as detection-isolation rates.

The possibility of a better prediction of the epidemic evolution can be enhanced by a more accurate knowledge of the transmission parameters and by a correct calibration of the model. Some of the parameters cannot be obtained by direct measurements. For instance, the detection rates are notoriously hard to be measured during an epidemic (see [6]). This leads to the necessity of calibrating the model by identification techniques using available measured data for the state. There are many recent papers dealing with the parameter estimation by various techniques using a best-fit approach, namely by finding the parameters that locally minimize the sum of the squares of the errors, for models with many compartments well evidenced during the SARS-CoV2 outbreak, see e.g., [2-4]. We also refer to the numerical simulations presented in [6] emphasizing the sensitivity of the predictions of the detection rates under various scenarios corresponding to the intervention policies. 


\subsection{Motivation and statement of the problem}

We see that if $l_{A}=l_{I}=\mu_{L}=0$, the class of isolated individuals disappear and so all asymptomatic and symptomatic remain in circulation. Thus, the class $L$ is relevant as a control class for the disease containment and a better isolation action can be done via the coefficients $l_{A}$ and $l_{I}$, within a time interval $[0, T]$. These parameters standing for the rates of isolation, as explained before, cannot be precisely identified by direct observations, since there is not a reliable control on the confirmed but unhospitalized people. Thus, we focus here only on the determination of the parameters $l_{A}$ and $l_{I}$ using the measured confirmed and recovered cases.

Let us assume a certain epidemiological evolution within the interval $[0, T]$, expressed by known initial data, the observed values $L_{o b s}(t)$ and $R_{o b s}(t)$, for the classes $L$ and $R$, and known system parameters, except for $l_{A}$ and $l_{I}$. In view of a correct analysis of the epidemic evolution a basic requirement is to find the parameters $l_{A}$ and $l_{I}$ which determined the components $L$ and $R$ of the system to evolve according the observed behaviors $L_{o b s}(t)$ and $R_{o b s}(t)$ within the interval [0,T].

From the mathematical point of view this means to minimize the functional

$$
\begin{aligned}
J\left(l_{A}, l_{I}\right)= & \frac{\alpha_{L}}{2} \int_{0}^{T}\left(L(t)-L_{o b s}(t)\right)^{2} d t+\frac{\alpha_{R}}{2} \int_{0}^{T}\left(R(t)-R_{o b s}(t)\right)^{2} d t \\
& +\frac{\alpha_{1}}{2} \int_{0}^{T}\left(l_{A}^{2}(t)+l_{I}^{2}(t)\right) d t,
\end{aligned}
$$

subject to system (1.1)-(1.6), (1.7). Here, $\alpha_{L}, \alpha_{R}$ and $\alpha_{1}$ are positive constants and

$$
L_{o b s}, R_{o b s} \in L^{\infty}(0, T), L_{o b s}(t) \in[0, N], R_{o b s}(t) \in[0, N] \text { a.e. on }(0, T) .
$$

As a component of the state system, $L(t) \in[0, N]$. However, as is it unlikely to test or isolate all the population at the same time, it is reasonable to assume that $L(t)$ does not reach the value $N$, but is bounded by a certain threshold less than $N$. In particular, the maximum value of $L$ cannot exceed the number of tests done at time $t$, and we denote this time dependent threshold the testing capacity $L_{\text {test }}(t)$. Thus, we minimize the functional along a constrained state $L(t) \in\left[0, L_{\text {test }}(t)\right]$, that is

$$
\begin{aligned}
\operatorname{Minimize} & \left\{J\left(l_{A}, l_{I}\right) ; l_{A}(t) \in[0,1], l_{I}(t) \in[0,1] \text { a.a. } t \in(0, T),\right. \\
& \left.0 \leq L(t) \leq L_{\text {test }}(t)\right\}
\end{aligned}
$$

subject to (1.1)-(1.7). We assume that $L_{\text {test }} \in C([0, T])$ and assume

$$
L_{0} \leq L_{\min }:=\min _{t \in[0, T]}\left\{L_{t e s t}(t)\right\}
$$

We note that the lower bound $L \geq 0$ is not actually a constraint because this follows from a property which will be proved for the solution to the state system. 
In the sequel, we assume the following conditions for the coefficients of the system:

$$
\begin{aligned}
& \beta_{I}, \beta_{A} \in L^{\infty}(0, \infty), \beta_{I}(t) \geq 0, \beta_{A}(t) \geq 0, \text { a.a. } t \geq 0, \\
& \sigma, \mu_{A}, \mu_{I}, \mu_{L}, \xi \geq 0,
\end{aligned}
$$

and denote

$$
\left.k_{1}(t):=\sigma+\mu_{A}+l_{A}(t), k_{2}(t):=\mu_{I}+l_{I}(t), k_{1}>0, k_{2}>0 \text {, a.e on } 0, T\right) .
$$

We approach problem $(P)$ by an optimal control technique. In Sect. 2, after proving the existence and uniqueness of the solution to the state system (1.1)-(1.6), we show that there exists at least a solution $\left(l_{A}^{*}, l_{I}^{*}\right)$ to problem $(P)$ in Proposition 2.2. For this problem the optimality conditions cannot be directly calculated since the cost functional is not regular because of the constraint. So, one introduces, in Sect. 3.1, an approximating problem $\left(P_{\varepsilon}\right)$ indexed along a positive parameter $\varepsilon$, which contains an appropriate penalized term replacing the state constraint. The convergence of a sequence of solutions to $\left(P_{\varepsilon}\right)$ precisely to a certain chosen solution to $(P)$ is proved in Proposition 3.2 and the approximating optimality conditions are provided in Proposition 3.3. Certain particular arguments and calculations are led to find appropriate estimates for the solution to the approximating dual system in Proposition 4.1. Actually, they ensure the fact that one component is a bounded in the class of BV (bounded variations) functions. Relying on these, local conditions of optimality for problem $(P)$ are obtained by passing to the limit in the approximating optimality condition system, in Theorem 4.3.

We note that $(P)$, with the state constraint $L(t) \in\left[0, L_{\text {test }}\right]$, requires a more challenging treatment involving a generalization of the basic duality theory to problems in which the trajectories are not absolutely continuous, but only with bounded variation. As a matter of fact, this is a typical phenomenon for state constraint optimal control problems (see [8]). Actually, the maximum principle (the first order conditions of optimality) leads to a singular dual backward system with a generalized solution in the sense of measure, which allows for jumps caused by the presence of the state constraint in the primal problem. As far as we know, this approach of identifying the parameters of a SARS-CoV-2 epidemic considering a state constraint imposed in connection with the real actions undertaken for the disease containment, is not found in the literature devoted to epidemics and represents a novelty in this paper.

\section{Problem $(\boldsymbol{P})$}

We begin with the proof of the well-posedness of the state system. Its solution will be sometimes denoted by $X:=(S, A, I, L, R)$.

By a solution to (1.1)-(1.6) on $[0, T]$ we mean an $\mathbb{R}^{5}$-valued absolutely continuous function $X=(S, A, I, L, R)$ on $[0, T]$ which satisfies (1.1)-(1.6) a.e. on $(0, T)$. 
Proposition 2.1 Let $T>0, l_{A}(t) \in[0,1], l_{I}(t) \in[0,1]$ a.e. on $(0, T)$. Let $X_{0}=$ $\left(S_{0}, A_{0}, I_{0}, L_{0}, R_{0}\right)$ with each component belonging to $[0, N]$. The state system (1.1)(1.7) has a unique global solution $X=(S, A, I, L, R) \in\left(W^{1, \infty}(0, T)\right)^{5}$ and each component belongs to $[0, N]$. The solution is continuous with respect to the data $\left(l_{A}, l_{I}\right)$.

Proof In system (1.1)-(1.6) we apply the Banach fixed point theorem, using the set

$$
\mathcal{M}=\{A \in C([0, T]) ; 0 \leq A(t) \leq N \text { for all } t \in[0, T]\}
$$

Let us pick $a \in \mathcal{M}$ and fix it in the system

$$
\begin{aligned}
& S^{\prime}(t)=-\beta_{I}(t) S(t) I(t)-\beta_{A}(t) S(t) a(t)+\xi R(t), \\
& A^{\prime}(t)=\beta_{I}(t) S(t) I(t)+\beta_{A}(t) S(t) a(t)-k_{1}(t) A(t), \\
& I^{\prime}(t)=\sigma a(t)-k_{2}(t) I(t), \\
& L^{\prime}(t)=l_{A}(t) a(t)+l_{I}(t) I(t)-\mu_{L} L(t), \\
& R^{\prime}(t)=\mu_{A} a(t)+\mu_{I} I(t)+\mu_{L} L(t)-\xi R(t),
\end{aligned}
$$

with the initial conditions (1.6). We define $\Psi: \mathcal{M} \rightarrow C([0, T])$ by $\Psi(a)=A$ where $(S, A, I, L, R)$ still denotes the solution to (2.1)-(2.5), (1.6) and show that $\Psi(\mathcal{M}) \subset \mathcal{M}$ and that $\Psi$ is a contraction on $\mathcal{M}$. Applying the formula of variation of constants in (2.3)

$$
I(t)=I_{0} e^{-\int_{0}^{t} k_{2}(\tau) d \tau}+\sigma \int_{0}^{t} e^{-\int_{s}^{t} k_{2}(\tau) d \tau} a(s) d s, t \in[0, T],
$$

we deduce that $I \in C([0, T]) \cap W^{1, \infty}(0, T)$. Moreover, $I(t) \geq 0$ for all $t \geq 0$. Applying successively the same formula in (2.4), (2.5), (2.1) and (2.2) we obtain that $L, R, S, A \in C([0, T]) \cap W^{1, \infty}(0, T)$ and each of them is nonnegative. By (1.7) each component is less or equal to $N$. Thus, $\Psi(\mathcal{M}) \subset \mathcal{M}$. It remains to prove that $\Psi$ is a contraction. Let us take two solutions to (2.1)-(2.5) $(S, A, I, L, R)$ and $(\bar{S}, \bar{A}, \bar{I}, \bar{L}, \bar{R})$ corresponding to $a$ and $\bar{a}$ respectively, with the same initial condition. By calculating $(I-\bar{I})(t), \ldots(S-\bar{S})(t),(A-\bar{A})(t)$ by each corresponding equation we obtain

$$
\sup _{t \in[0, T]}|(A-\bar{A})(t)| \leq C_{1}(T) \sup _{t \in[0, T]}|(a-\bar{a})(t)|
$$

where $C_{1}(T)$ is a polynomial in $T$ with coefficients consisting in sums of the constant systems parameters and the $L^{\infty}$-norms of the time dependent system parameters. This shows that $\Psi$ is a contraction for small $T$, that is we obtain a local solution. Since all solution components are bounded by $N$ it follows that the solution is global (see e.g., [9], p. 41, Theorem 2.15). By a similar calculation one can easily prove the continuity with respect to $l_{A}$ and $l_{I}$.

Proposition 2.2 Problem $(P)$ has at least one solution $\left(l_{A}^{*}, l_{I}^{*}\right)$. 
Proof It is obvious that an admissible pair exists. For example, for $l_{A}=l_{I}=0$, by (1.4) and (1.9) we get $L(t) \leq L_{0} \leq L_{\text {test }}(t)$ and $J\left(l_{A}, l_{I}\right)<\infty$. Let $d:=\inf J\left(l_{A}, l_{I}\right) \geq 0$ and consider a minimizing sequence $\left(l_{A}^{n}, l_{I}^{n}\right)_{n}$, satisfying the restrictions in $(P)$. The minimizing sequence also satisfies

$$
d \leq J\left(l_{A}^{n}, l_{I}^{n}\right) \leq d+\frac{1}{n}, \text { for } n \geq 1
$$

This implies that $l_{A}^{n} \rightarrow l_{A}^{*}$ and $l_{I}^{n} \rightarrow l_{I}^{*}$ weak $^{*}$ in $L^{\infty}(0, T)$, as $n \rightarrow \infty$ and $l_{A}^{*}$, $l_{I}^{*} \in[0,1]$ a.e. We denote by $X^{n}$ the solution to the state system corresponding to the minimizing sequence. By Proposition 2.1 this solution exists and is bounded in $\left(W^{1, \infty}(0, T)\right)^{5}$. Each solution component belongs to $[0, N]$ and $L^{n}(t) \leq L_{\text {test }}(t)$. Then, on a subsequence, $X^{n} \rightarrow X^{*},\left(X^{n}\right)^{\prime} \rightarrow\left(X^{*}\right)^{\prime}$ weak* in $\left(W^{1, \infty}(0, T)\right)^{5}$. By Arzelà theorem it follows that $X^{n} \rightarrow X^{*}$ uniformly in $[0, T]$, and so $X^{n}(0) \rightarrow$ $X^{*}(0)=X_{0}$ and $X^{n}(T) \rightarrow X^{*}(T)$. By passing to the limit in (1.1)-(1.6) we deduce that $\left(X^{*}\right)^{\prime}(t)=\lim _{n \rightarrow \infty}\left(X^{n}\right)^{\prime}(t)$, whence it follows that $X^{*}$ is the solution to (1.1)(1.6) corresponding to $l_{A}^{*}, l_{I}^{*}$. Moreover, $L^{*}(t) \leq L_{\text {test }}(t)$ for all $t \in[0, T]$. Finally, by using the weakly lower semicontinuity of the norms we get $\lim _{n \rightarrow \infty} J\left(l_{A}^{n}, l_{I}^{n}\right)=$ $J\left(l_{A}^{*}, l_{I}^{*}\right)$ and so $\left(l_{A}^{*}, l_{I}^{*}\right)$ turns out to be optimal in $(P)$.

\section{The Approximating Problem $\left(\boldsymbol{P}_{\varepsilon}\right)$}

\subsection{Existence and Convergence of the Approximating Control}

Let $\varepsilon>0$ and let $\left(l_{A}^{*}, l_{I}^{*}\right)$ be optimal in $(P)$. We introduce the adapted approximating cost functional

$$
\begin{aligned}
J_{\varepsilon}\left(l_{A}, l_{I}\right)= & \frac{\alpha_{L}}{2} \int_{0}^{T}\left(L(t)-L_{o b s}(t)\right)^{2} d t+\frac{\alpha_{R}}{2} \int_{0}^{T}\left(R(t)-R_{o b s}(t)\right)^{2} d t \\
& +\frac{\alpha_{1}}{2} \int_{0}^{T}\left(l_{A}^{2}(t)+l_{I}^{2}(t)\right) d t+\frac{1}{2} \int_{0}^{T}\left\{\left(l_{A}(t)-l_{A}^{*}(t)\right)^{2}+\left(l_{I}(t)-l_{I}^{*}(t)\right)^{2}\right\} d t \\
& +\frac{\alpha_{2}}{2 \varepsilon} \int_{0}^{T}\left(\left(L(t)-L_{\text {test }}(t)\right)^{+}\right)^{2} d t
\end{aligned}
$$

and study the following approximating problem $\left(P_{\varepsilon}\right)$,

$\operatorname{Minimize}\left\{J_{\varepsilon}\left(l_{A}, l_{I}\right) ; l_{A} \in[0,1], l_{I} \in[0,1]\right.$ a.e. on $\left.(0, T)\right\}$

subject to (1.1)-(1.7).

We observe that the state constraint in $(P)$ is replaced here by the penalization of the $L^{2}$-norm of the positive part of $\left(L(t)-L_{t e s t}(t)\right)$, where $\alpha_{2}>0$. The last two penalization terms in (3.1) will ensure the convergence of the approximating solution to the chosen optimal controller $\left(l_{A}^{*}, l_{I}^{*}\right)$ in $(P)$. 
Proposition 3.1 Problem $\left(P_{\varepsilon}\right)$ has at least one solution, $\left(l_{A, \varepsilon}^{*}, l_{I, \varepsilon}^{*}\right)$ with the corresponding state $X_{\varepsilon}^{*} \in\left(W^{1, \infty}(0, T)\right)^{5}$, having the components in $[0, N]$.

After observing that there is at least an admissible triplet (let it be an optimal one in $(P)$ ), the proof is led then exactly as in Proposition 2.2, using in addition the continuity of the function $L \rightarrow L^{+}$.

Proposition 3.2 Let $\left\{\left(l_{A}^{*}, l_{I}^{*}\right), X^{*}\right\}$ and $\left\{\left(l_{A, \varepsilon}^{*}, l_{I, \varepsilon}^{*}\right), X_{\varepsilon}^{*}\right\}_{\varepsilon}$ be optimal in $(P)$ and in $\left(P_{\varepsilon}\right)$, respectively. Then,

$$
\begin{aligned}
& l_{A, \varepsilon}^{*} \rightarrow l_{A}^{*}, l_{I, \varepsilon}^{*} \rightarrow l_{I}^{*} \text { weak }^{*} \text { in } L^{\infty}(0, T) \text { and strongly in } L^{2}(0, T) \text {, as } \varepsilon \rightarrow 0, \\
& X_{\varepsilon} \rightarrow X^{*} \text { weak }^{*} \text { in }\left(W^{1, \infty}(0, T)\right)^{5} \text { and uniformly in }[0, T] \text {, as } \varepsilon \rightarrow 0
\end{aligned}
$$

Proof If $\left(l_{A, \varepsilon}^{*}, l_{I, \varepsilon}^{*}\right)$ is optimal, then $J_{\varepsilon}\left(l_{A, \varepsilon}^{*}, l_{I, \varepsilon}^{*}\right) \leq J_{\varepsilon}\left(l_{A}, l_{I}\right)$, for all $\left(l_{A}, l_{I}\right)$ satisfying the constraints in $\left(P_{\varepsilon}\right)$. In particular, we can set $\left(l_{A}, l_{I}\right)=\left(l_{A}^{*}, l_{I}^{*}\right)$ a.e. on $(0, T)$ which is the optimal triplet chosen in $(P)$ and then the previous inequality becomes

$$
\begin{aligned}
J_{\varepsilon}\left(l_{A, \varepsilon}^{*}, l_{I, \varepsilon}^{*}\right)= & \frac{\alpha_{L}}{2} \int_{0}^{T}\left(L_{\varepsilon}^{*}(t)-L_{o b s}(t)\right)^{2} d t+\frac{\alpha_{R}}{2} \int_{0}^{T}\left(R_{\varepsilon}^{*}(t)-R_{o b s}(t)\right)^{2} d t \\
& +\frac{\alpha_{1}}{2} \int_{0}^{T}\left(\left(l_{A, \varepsilon}^{*}(t)\right)^{2}+\left(l_{I, \varepsilon}^{*}(t)\right)^{2}\right) d t+\frac{1}{2} \int_{0}^{T}\left(l_{A, \varepsilon}^{*}(t)-l_{A}^{*}(t)\right)^{2} d t \\
& +\frac{1}{2} \int_{0}^{T}\left(l_{I, \varepsilon}^{*}(t)-l_{I}^{*}(t)\right)^{2} d t+\frac{\alpha_{2}}{2 \varepsilon} \int_{0}^{T}\left(\left(L_{\varepsilon}^{*}(t)-L_{\text {test }}(t)\right)^{+}\right)^{2}(t) d t \\
\leq & J_{\varepsilon}\left(l_{A}^{*}, l_{I}^{*}\right)=\frac{\alpha_{L}}{2} \int_{0}^{T}\left(L^{*}(t)-L_{o b s}(t)\right)^{2} d t+\frac{\alpha_{R}}{2} \int_{0}^{T}\left(R^{*}(t)-R_{\text {obs }}(t)\right)^{2} d t \\
& +\frac{\alpha_{1}}{2} \int_{0}^{T}\left(l_{A}^{* 2}(t)+l_{I}^{* 2}(t)\right) d t
\end{aligned}
$$

because the optimal $L^{*} \leq L_{\text {test }}$ in $(P)$. Thus, the left-hand side is bounded and we deduce that, on a subsequence, $l_{A, \varepsilon}^{*} \rightarrow l_{A}^{* *}, l_{I, \varepsilon}^{*} \rightarrow l_{I}^{* *}$ weakly in $L^{2}(0, T)$, weak* in $L^{\infty}(0, T)$ as $\varepsilon \rightarrow 0$, and $l_{A}^{* *}, l_{I}^{* *} \in[0,1]$. As in Proposition 2.2, we infer that $X_{\varepsilon} \rightarrow X^{* *}$ weak $^{*}$ in $\left(W^{1, \infty}(0, T)\right)^{5}$ and uniformly in $[0, T]$. By (3.4) we can write

$$
\frac{\alpha_{2}}{2 \varepsilon} \int_{0}^{T}\left(\left(L_{\varepsilon}^{*}-L_{t e s t}\right)^{+}(t)\right)^{2} d t \leq \text { constant }
$$

which implies that $\int_{0}^{T}\left(\left(L_{\varepsilon}^{*}-L_{\text {test }}\right)^{+}(t)\right)^{2} d t \rightarrow 0$ and so, $\left(L_{\varepsilon}^{*}-L_{\text {test }}\right)^{+} \rightarrow 0$ strongly in $L^{2}(0, T)$. On the other hand, $\left(L_{\varepsilon}^{*}-L_{\text {test }}\right)^{+}(t) \rightarrow\left(L^{* *}-L_{\text {test }}\right)^{+}(t)$ for all $t \in[0, T]$, so that $L^{* *}(t) \leq L_{\text {test }}(t)$. In the inequality (3.4) without the last three terms on the left-hand side we pass to the limit using the weakly lower semicontinuity of the norms and deduce that $J\left(l_{A}^{* *}, l_{I}^{* *}\right) \leq J\left(l_{A}^{*}, l_{I}^{*}\right)$, hence $\left(l_{A}^{* *}, l_{I}^{* *}\right)$ is optimal in $(P)$, too. Moreover, still by (3.4) we easily infer that $l_{A, \varepsilon}^{*} \rightarrow l_{A}^{*}$ and $l_{I, \varepsilon}^{*} \rightarrow l_{I}^{*}$ strongly in $L^{2}(0, T)$, hence $\left(l_{A}^{* *}, l_{I}^{* *}\right)=\left(l_{A}^{*}, l_{I}^{*}\right)$ a.e. This ends the proof . 


\subsection{The Approximating Optimality Conditions}

Let $\lambda>0$ and set the variations

$$
\begin{aligned}
& l_{A, \varepsilon}^{\lambda}=l_{A, \varepsilon}^{*}+\lambda \omega_{A}, \omega_{A}=\tilde{l_{A}}-l_{A, \varepsilon}^{*}, \tilde{l_{A}}(t) \in(0,1), \text { a.e. } t \in(0,1) \\
& l_{I, \varepsilon}^{\lambda}=l_{I, \varepsilon}^{*}+\lambda \omega_{I}, \omega_{I}=\tilde{l}_{I}-l_{I, \varepsilon}^{*}, \tilde{l_{I}}(t) \in[0,1], \text { a.e. } t \in(0,1) .
\end{aligned}
$$

Let us denote

$$
x_{\varepsilon}^{\lambda}:=\frac{X_{\varepsilon}^{l_{A, \varepsilon}^{\lambda}, l_{I, \varepsilon}^{\lambda}}-X_{\varepsilon}^{*}}{\lambda}
$$

where $X_{\varepsilon}^{l_{A, \varepsilon}^{\lambda}, l_{I, \varepsilon}^{\lambda}}$ is the solution to (1.1)-(1.7) corresponding to $\left(l_{A, \varepsilon}^{\lambda}, l_{I, \varepsilon}^{\lambda}\right)$, and $X_{\varepsilon}^{*}$ is the optimal state corresponding to $\left(l_{A, \varepsilon}^{*}, l_{I, \varepsilon}^{*}\right)$.

We introduce the linearized system for problem $(P)$

$$
\begin{aligned}
s^{\prime} & =-k_{0, \varepsilon} s-\beta_{I} S_{\varepsilon}^{*} i-\beta_{A} S_{\varepsilon}^{*} a+\xi r, \\
a^{\prime} & =k_{0, \varepsilon} s+\beta_{I}^{*} S_{\varepsilon}^{*} i+k_{3, \varepsilon} a-\omega_{A} A_{\varepsilon}^{*}, \\
i^{\prime} & =\sigma a-k_{2, \varepsilon} i-\omega_{I} I_{\varepsilon}^{*}, \\
l^{\prime} & =l_{A, \varepsilon}^{*} a+l_{I, \varepsilon}^{*} i-\mu_{L} l+\omega_{A} A_{\varepsilon}^{*}+\omega_{I} I_{\varepsilon}^{*}, \\
r^{\prime} & =\mu_{A} a+\mu_{I} i+\mu_{L} l-\xi r,
\end{aligned}
$$

for a.a. $t>0$, with the initial condition

$$
s(0)=0, a(0)=0, i(0)=0, l(0)=0, r(0)=0,
$$

where

$$
\begin{aligned}
& k_{0, \varepsilon}=\beta_{A} A_{\varepsilon}^{*}+\beta_{I} I_{\varepsilon}^{*}, k_{1, \varepsilon}:=\sigma+\mu_{A}+l_{A, \varepsilon}^{*}, \\
& k_{2, \varepsilon}=\mu_{I}+l_{I, \varepsilon}^{*}, k_{3, \varepsilon}:=\beta_{A} S_{\varepsilon}^{*}-k_{1, \varepsilon} .
\end{aligned}
$$

First, by the known results for linear systems we infer that (3.5) has a unique global solution $x=(s, a, i, l, r) \in\left(W^{1, \infty}(0, T)\right)^{5}$.

By a direct calculation, using the continuity with respect to the data of the solution to the state system it can be easily proved that $x_{\varepsilon}^{\lambda} \rightarrow x_{\varepsilon}:=(s, a, i, l, r)$ strongly in $C([0, T])$, as $\lambda \rightarrow 0$, so that (3.5) stands for the system in variations.

We introduce the backward dual system for the variables $\left(p_{\varepsilon}, q_{\varepsilon}, d_{\varepsilon}, e_{\varepsilon}, f_{\varepsilon}\right)$ as

$$
\begin{aligned}
& p_{\varepsilon}^{\prime}-k_{0, \varepsilon} p_{\varepsilon}+k_{0, \varepsilon} q_{\varepsilon}=0, \\
& q_{\varepsilon}^{\prime}-\beta_{A} S_{\varepsilon}^{*} p_{\varepsilon}+k_{3, \varepsilon} q_{\varepsilon}+\sigma d_{\varepsilon}+l_{A, \varepsilon}^{*} e_{\varepsilon}+\mu_{A} f_{\varepsilon}=0, \\
& d_{\varepsilon}^{\prime}-\beta_{I} S_{\varepsilon}^{*} p_{\varepsilon}+\beta_{I} S_{\varepsilon}^{*} q_{\varepsilon}-k_{2, \varepsilon} d_{\varepsilon}+l_{I, \varepsilon}^{*} e_{\varepsilon}+\mu_{I} f_{\varepsilon}=0, \\
& e_{\varepsilon}^{\prime}-\mu_{L} e_{\varepsilon}+\mu_{L} f_{\varepsilon}+\frac{\alpha_{2}}{\varepsilon}\left(L_{\varepsilon}^{*}-L_{t e s t}\right)^{+}=-\alpha_{L}\left(L_{\varepsilon}^{*}-L_{o b s}\right),
\end{aligned}
$$




$$
f_{\varepsilon}^{\prime}+\xi p_{\varepsilon}-\xi f_{\varepsilon}=-\alpha_{R}\left(R_{\varepsilon}^{*}-L_{o b s}\right)
$$

for a.a. $t>0$, with the final conditions

$$
p_{\varepsilon}(T)=0, q_{\varepsilon}(T)=0, d_{\varepsilon}(T)=0, e_{\varepsilon}(T)=0, f_{\varepsilon}(T)=0 .
$$

The linear system (3.8)-(3.13) has a unique global solution $\left(p_{\varepsilon}, q_{\varepsilon}, d_{\varepsilon}, e_{\varepsilon}, f_{\varepsilon}\right) \in$ $\left(W^{1, \infty}(0, T)\right)^{5}$, for each $\varepsilon>0$.

Let $N_{[0,1]}(z)$ be the normal cone to the set $[0,1]$,

$$
N_{[0,1]}(z)=\left\{\begin{array}{l}
\mathbb{R}^{-}, \text {if } z=0 \\
0, \text { if } z \in(0,1), \\
\mathbb{R}^{+}, \text {if } z=1
\end{array}\right.
$$

We recall that the projection of a point $z$ on a set $K \subset \mathbb{R}^{d}, d \in \mathbb{N}$, is defined by

$$
P_{K}(z):=\left(I_{d}+\kappa \partial I_{k}\right)^{-1}(z)
$$

for all $k>0, I_{d}$ being the identity operator.

Proposition 3.3 Let $\left(l_{A, \varepsilon}^{*}, l_{I, \varepsilon}^{*}\right)$ be optimal in $\left(P_{\varepsilon}\right)$ with the state $X_{\varepsilon}^{*}$. Then,

$$
\begin{aligned}
& l_{A, \varepsilon}^{*}(t)=P_{[0,1]}\left(\frac{1}{\alpha_{1}+1}\left(A_{\varepsilon}^{*}\left(q_{\varepsilon}-e_{\varepsilon}\right)+l_{A}^{*}\right)(t)\right), \text { a.e. } t \in(0, T), \\
& l_{I, \varepsilon}^{*}(t)=P_{[0,1]}\left(\frac{1}{\alpha_{1}+1}\left(I_{\varepsilon}^{*}\left(d_{\varepsilon}-e_{\varepsilon}\right)+l_{I}^{*}\right)(t)\right), \text { a.e. } t \in(0, T),
\end{aligned}
$$

where $\left(p_{\varepsilon}, q_{\varepsilon}, d_{\varepsilon}, e_{\varepsilon}, f_{\varepsilon}\right)$ is the solution to the backward dual system (3.8)-(3.13).

Proof Let us multiply the equations for $s, a, i, l, r$ in (3.5) by $p_{\varepsilon}, q_{\varepsilon}, d_{\varepsilon}, e_{\varepsilon}, f_{\varepsilon}$, respectively and integrate over $(0, T)$. By integrating by parts and taking into account the dual system and the initial conditions in the system in variations, we obtain

$$
\begin{aligned}
& \alpha_{L} \int_{0}^{T}\left(L_{\varepsilon}^{*}-L_{o b s}\right) l d t+\alpha_{R} \int_{0}^{T}\left(R_{\varepsilon}^{*}-R_{o b s}\right) r d t+\frac{\alpha_{2}}{\varepsilon} \int_{0}^{T}\left(L_{\varepsilon}^{*}-L_{t e s t}\right)^{+} l d t \\
& \quad=\int_{0}^{T}\left(\omega_{A} A_{\varepsilon}^{*}\left(e_{\varepsilon}-q_{\varepsilon}\right)+\omega_{I} I_{\varepsilon}^{*}\left(e_{\varepsilon}-d_{\varepsilon}\right)\right) d t
\end{aligned}
$$

On the other hand, for $\left(l_{A, \varepsilon}^{*}, l_{I, \varepsilon}^{*}\right)$ optimal in $\left(P_{\varepsilon}\right)$ we can write

$$
J_{\varepsilon}\left(l_{A, \varepsilon}^{\lambda}, l_{I, \varepsilon}^{\lambda}\right) \geq J_{\varepsilon}\left(l_{A, \varepsilon}^{*}, l_{I, \varepsilon}^{*}\right) .
$$

By replacing the expression of the cost functional $J_{\mathcal{E}}$, performing some algebra, dividing by $\lambda$ and passing to the limit as $\lambda \rightarrow 0$ we obtain 


$$
\begin{aligned}
& \alpha_{L} \int_{0}^{T}\left(L_{\varepsilon}^{*}-L_{o b s}\right) l d t+\alpha_{R} \int_{0}^{T}\left(R_{\varepsilon}^{*}-R_{o b s}\right) r d t+\frac{\alpha_{2}}{\varepsilon} \int_{0}^{T}\left(L_{\varepsilon}^{*}-L_{t e s t}\right)^{+} l d t \\
& \quad+\alpha_{1} \int_{0}^{T}\left(l_{A, \varepsilon}^{*} \omega_{A}+l_{I, \varepsilon}^{*} \omega_{I}\right) d t+\int_{0}^{T}\left\{\left(l_{A, \varepsilon}^{*}-l_{A}^{*}\right) \omega_{A}+\left(l_{I, \varepsilon}^{*}-l_{I}^{*}\right) \omega_{I}\right\} d t \geq 0 .
\end{aligned}
$$

By comparison with (3.15) we deduce that

$$
\begin{aligned}
& \int_{0}^{T} \omega_{A} A_{\varepsilon}^{*}\left(e_{\varepsilon}-q_{\varepsilon}\right) d t+\int_{0}^{T}\left(\left(\alpha_{1}+1\right) l_{A, \varepsilon}^{*}-l_{A}^{*}\right) \omega_{A} d t \\
& +\int_{0}^{T} \omega_{I} I_{\varepsilon}^{*}\left(e_{\varepsilon}-d_{\varepsilon}\right) d t+\int_{0}^{T}\left(\left(\alpha_{1}+1\right) l_{I, \varepsilon}^{*}-l_{I}^{*}\right) \omega_{I} d t \geq 0 .
\end{aligned}
$$

We recall the setting of $\omega_{A}$ and $\omega_{I}$ and choose, in particular, $\tilde{l_{A}}=l_{A, \varepsilon}^{*}$, meaning that we keep $l_{A, \varepsilon}^{*}$ fixed and give a variation only to $l_{I, \varepsilon}^{*}$. Then, (3.17) yields

$$
\int_{0}^{T}\left(-I_{\varepsilon}^{*}\left(e_{\varepsilon}-d_{\varepsilon}\right)-\left(\alpha_{1}+1\right) l_{I, \varepsilon}^{*}+l_{I}^{*}\right)\left(l_{I, \varepsilon}^{*}-\tilde{l}_{I}\right) d t \geq 0
$$

for $\tilde{l}_{I}(t) \in[0,1]$ a.e. $t \in(0, T)$, which implies

$$
\left(-I_{\varepsilon}^{*}\left(e_{\varepsilon}-d_{\varepsilon}\right)-\left(\alpha_{1}+1\right) l_{I, \varepsilon}^{*}+l_{I}^{*}\right)(t) \in N_{[0,1]}\left(l_{I, \varepsilon}^{*}(t)\right) \text {, a.e. } t \in(0, T),
$$

whence we have the second relation in (3.14).

Then, let us set $\widetilde{l_{I}}=l_{I, \varepsilon}^{*}$. By (3.17) we obtain that

$$
\left(-A_{\varepsilon}^{*}\left(e_{\varepsilon}-q_{\varepsilon}\right)-\left(\alpha_{1}+1\right) l_{A, \varepsilon}^{*}+l_{A}^{*}\right)(t) \in N_{[0,1]}\left(l_{A, \varepsilon}^{*}(t)\right),
$$

for $\tilde{l_{A}}(t) \in[0,1]$ a.e. $t \in(0, T)$, which yields the first relation in (3.14).

\section{The Optimality Conditions for Problem (P)}

We begin by proving the boundedness of the solution to the dual system.

Proposition 4.1 There exists $T_{l o c}$, such that for $T<T_{\text {loc }}$ we have

$$
\begin{array}{ll}
\left|e_{\varepsilon}(t)\right|+\int_{0}^{T}\left|e_{\varepsilon}^{\prime}(t)\right| d t \leq C, & \text { for all } t \in[0, T], \\
\left|p_{\varepsilon}(t)\right|+\left|q_{\varepsilon}(t)\right|+\left|d_{\varepsilon}(t)\right|+\left|f_{\varepsilon}(t)\right| \leq C, & \text { for all } t \in[0, T],
\end{array}
$$

with $C$ independent of $\varepsilon$. 
Proof By (1.9) we have $L_{\min }=\min _{t \in[0, T]}\left\{L_{\text {test }}(t)\right\}$ and recall that $L_{\min } \geq L_{0}$. Let us choose $y_{1} \in\left(0, L_{\min }\right), \rho>0$ and $\theta \in \mathbb{R}$, with $|\theta|=1$ such that $y_{1}+\rho \theta \in\left(0, L_{\text {min }}\right)$. This can be equivalently written $0 \leq y_{1} \pm \rho<L_{\min }$ and holds if

$$
0<\rho<\min \left\{y_{1}, L_{\min }-y_{1}\right\}
$$

The values $y_{1}$ and $\rho$ are at our free choice and different situations for the positions of $L_{0}, L_{\min }$ and $y_{1}$ will be discussed later, at the appropriate place.

For the moment, let us multiply (3.11) by $\left(L_{\varepsilon}^{*}-\left(y_{1}+\rho \theta\right)\right)$ and integrate over $(0, T)$. We have

$$
\begin{aligned}
& \int_{0}^{T}\left(L_{\varepsilon}^{*}-y_{1}\right) e_{\varepsilon}^{\prime} d t-\int_{0}^{T} \rho \theta e_{\varepsilon}^{\prime} d t+\frac{\alpha_{2}}{\varepsilon} \int_{0}^{T}\left(L_{\varepsilon}^{*}-L_{t e s t}\right)^{+}\left(L_{\varepsilon}^{*}-y_{1}-\rho \theta\right) d t \\
& \quad=\int_{0}^{T}\left(\mu_{L} e_{\varepsilon}-\mu_{L} f_{\varepsilon}\right)\left(L_{\varepsilon}^{*}-y_{1}-\rho \theta\right) d t-\int_{0}^{T} \alpha_{L}\left(L_{\varepsilon}^{*}-L_{o b s}\right)\left(L_{\varepsilon}^{*}-y_{1}-\rho \theta\right) d t .
\end{aligned}
$$

Let us define $\varphi(y)=\frac{1}{2}\left(y^{+}\right)^{2}, \varphi: \mathbb{R} \rightarrow[0, \infty)$. Its subdifferential $\partial \varphi(y)=y^{+}$and $\varphi(y)-\varphi(z) \leq \partial \varphi(y)(y-z)$ for all $z \geq 0$. In the second term on the left-hand side of (4.4) we set

$$
\theta:=-\frac{e_{\varepsilon}^{\prime}}{\left|e_{\varepsilon}^{\prime}\right|} 1_{\left\{t ; e_{\varepsilon}^{\prime}(t) \neq 0\right\}},
$$

where $1_{\left\{t ; e_{\varepsilon}^{\prime}(t) \neq 0\right\}}$ is the characteristic function of the set indicated as subscript. Using the relation for the subdifferential of $\varphi$ and integrating by parts the first term on the left-hand side of (4.4) we have

$$
\begin{aligned}
& \left(L_{\varepsilon}^{*}(T)-y_{1}\right) e_{\varepsilon}(T)-\left(L_{\varepsilon}^{*}(0)-y_{1}\right) e_{\varepsilon}(0)-\int_{0}^{T}\left(L_{\varepsilon}^{*}\right)^{\prime} e_{\varepsilon} d t+\rho \int_{0}^{T}\left|e_{\varepsilon}^{\prime}(t)\right| d t \\
& +\frac{\alpha_{2}}{2 \varepsilon} \int_{0}^{T}\left(\left(L_{\varepsilon}^{*}-L_{t e s t}\right)^{+}\right)^{2} d t-\frac{\alpha_{2}}{2 \varepsilon} \int_{0}^{T}\left(\left(\left(y_{1}+\rho \theta\right)-L_{t e s t}\right)^{+}\right)^{2} d t \\
& \quad \leq \int_{0}^{T}\left(\mu_{L} e_{\varepsilon}-\mu_{L} f_{\varepsilon}\right) L_{\varepsilon}^{*} d t-\int_{0}^{T}\left(\mu_{L} e_{\varepsilon}-\mu_{L} f_{\varepsilon}\right)\left(y_{1}+\rho \theta\right) d t \\
& -\alpha_{L} \int_{0}^{T}\left(L_{\varepsilon}^{*}-L_{o b s}\right)\left(L_{\varepsilon}^{*}-y_{1}-\rho \theta\right) d t .
\end{aligned}
$$

Since $y_{1}+\rho \theta<L_{\min } \leq L_{\text {test }}(t), e_{\varepsilon}(T)=0$, using Eq. (1.4) and making some rearrangements we are led to the relation

$$
\begin{gathered}
\rho \int_{0}^{T}\left|e_{\varepsilon}^{\prime}(t)\right| d t \leq\left|L_{0}-y_{1}\right|\left|e_{\varepsilon}(0)\right|+\int_{0}^{T}\left(e_{\varepsilon}\left(l_{A, \varepsilon}^{*} A_{\varepsilon}^{*}+l_{I, \varepsilon}^{*} I_{\varepsilon}^{*}\right)-\mu_{L} f_{\varepsilon} L_{\varepsilon}^{*}\right) d t \\
\quad-\int_{0}^{T}\left(\mu_{L} e_{\varepsilon}-\mu_{L} f_{\varepsilon}\right)\left(y_{1}+\rho \theta\right) d t+\alpha_{L} \int_{0}^{T}\left|L_{\varepsilon}^{*}-L_{o b s}\right|\left|L_{\varepsilon}^{*}-y_{1}-\rho \theta\right| d t
\end{gathered}
$$




$$
\left.\leq \int_{0}^{T}\left(\left|e_{\varepsilon}\right|+\mid f_{\varepsilon}\right) \mid\right)\left(l_{A, \varepsilon}^{*}\left|A_{\varepsilon}^{*}\right|+l_{I, \varepsilon}^{*}\left|I_{\varepsilon}^{*}\right|+\mu_{L}\left|L_{\varepsilon}^{*}\right|+\mu_{L} N\right) d t+4 \alpha_{L} N^{2} T
$$

because $L_{\varepsilon}^{*}, L_{o b s}$ and $\left|y_{1}+\rho \theta\right|$ are smaller than $N$. We denote

$$
E_{\varepsilon}:=\int_{0}^{T}\left|e_{\varepsilon}^{\prime}(t)\right| d t, F_{0}:=\left|L_{0}-y_{1}\right|
$$

and since $e_{\varepsilon}(T)=0$, we note that

$$
\left|e_{\varepsilon}(0)\right|=\left|\int_{0}^{T} e_{\varepsilon}^{\prime}(t) d t\right| \leq E_{\varepsilon},\left|e_{\varepsilon}(t)\right| \leq E_{\varepsilon} \text { for all } t \in[0, T] .
$$

Then, we have (because $l_{A, \varepsilon}^{*}, l_{I, \varepsilon}^{*} \leq 1$ )

$$
\left.\rho E_{\varepsilon} \leq F_{0} E_{\varepsilon}+2 \int_{0}^{T}\left(\left|e_{\varepsilon}\right|+\mid f_{\varepsilon}\right) \mid\right)\left(\mu_{L}+1\right) N d t+4 \alpha_{L} N^{2} T .
$$

Let us denote $\mathcal{S}_{\varepsilon}^{2}(t)=\left|p_{\varepsilon}(t)\right|^{2}+\left|q_{\varepsilon}(t)\right|^{2}+\left|d_{\varepsilon}(t)\right|^{2}+\left|f_{\varepsilon}(t)\right|^{2}$. We multiply Eqs. (3.8)-(3.10), (3.12) by $p_{\varepsilon}, q_{\varepsilon}, d_{\varepsilon}, f_{\varepsilon}$ respectively, integrate over $(t, T)$ and sum up. After some calculations, and a variable change $t \rightarrow T-t$, we obtain

$$
\begin{aligned}
\frac{1}{2} \mathcal{S}_{\varepsilon}^{2}(t) \leq & \int_{0}^{t} \digamma_{\varepsilon} \mathcal{S}_{\varepsilon}^{2}(\tau) d \tau+l_{A, \varepsilon}^{*} \int_{0}^{t}\left|e_{\varepsilon}\right|\left|q_{\varepsilon}\right| d \tau+l_{I, \varepsilon}^{*} \int_{0}^{t}\left|e_{\varepsilon}\right|\left|d_{\varepsilon}\right| d \tau \\
& +\alpha_{R} \int_{0}^{t}\left|R_{\varepsilon}^{*}-R_{o b s}\right|\left|f_{\varepsilon}\right| d \tau \\
\leq & \int_{0}^{t} \digamma_{\varepsilon} \mathcal{S}_{\varepsilon}^{2}(\tau) d \tau+l_{A, \varepsilon}^{* 2} \int_{0}^{t}\left|q_{\varepsilon}(\tau)\right|^{2} d \tau+l_{I, \varepsilon}^{* 2} \int_{0}^{t}\left|d_{\varepsilon}(\tau)\right|^{2} d \tau+2 \int_{0}^{t}\left|e_{\varepsilon}(\tau)\right|^{2} d \tau \\
& +\alpha_{R}^{2} \int_{0}^{t}\left|f_{\varepsilon}(\tau)\right|^{2} d \tau+\int_{0}^{t}\left(\left|R_{\varepsilon}^{*}(\tau)\right|+\left|R_{o b s}\right|\right)^{2} d \tau,
\end{aligned}
$$

whence we get

$$
\mathcal{S}_{\varepsilon}^{2}(t) \leq \int_{0}^{t} G_{\varepsilon} \mathcal{S}_{\varepsilon}^{2}(\tau) d \tau+4 E_{\varepsilon}^{2} T+8 N^{2} T, \text { for all } t \in[0, T]
$$

Here, $\digamma_{\varepsilon}$ consists in sums of the constant coefficients of the equations in the dual system plus the $L^{\infty}$-norms of the time dependent coefficients (or square coefficients) and $G_{\varepsilon}=2\left(\digamma_{\varepsilon}+l_{A, \varepsilon}^{* 2}+l_{I, \varepsilon}^{* 2}+\alpha_{R}^{2}\right) \leq C$, since all terms in $G_{\varepsilon}$ are bounded. Further, $C$ may denote several positive constants. By the Gronwall's lemma, the previous inequality yields the estimate $\mathcal{S}_{\varepsilon}^{2}(t) \leq\left(8 E_{\varepsilon}^{2} T+4 N^{2} T\right) e^{C t}$, implying

$$
\left|p_{\varepsilon}(t)\right|+\left|q_{\varepsilon}(t)\right|+\left|d_{\varepsilon}(t)\right|+\left|f_{\varepsilon}(t)\right| \leq 4\left(E_{\varepsilon}+N\right) \sqrt{T} e^{C T} .
$$


We go back to (4.7) and using (4.8) we write

$$
\begin{aligned}
\rho E_{\varepsilon} & \leq F_{0} E_{\varepsilon}+2\left(\mu_{L}+1\right) N \int_{0}^{T}\left(E_{\varepsilon}+4\left(E_{\varepsilon}+N\right) \sqrt{T} e^{C T}\right) d t+4 \alpha_{L} N^{2} T \\
& \leq F_{0} E_{\varepsilon}+2\left(\mu_{L}+1\right) N\left(1+4 \sqrt{T} e^{C T}\right) T E_{\varepsilon}+\left(8\left(\mu_{L}+1\right) \sqrt{T} e^{C T}+4 \alpha_{L}\right) N^{2} T .
\end{aligned}
$$

This implies

$$
\begin{aligned}
& E_{\varepsilon}\left(\rho-F_{0}-2\left(\mu_{L}+1\right) N\left(1+4 \sqrt{T} e^{C T}\right) T\right) \\
& \leq 4\left(2\left(\mu_{L}+1\right) \sqrt{T} e^{C T}+\alpha_{L}\right) N^{2} T .
\end{aligned}
$$

We have to check if the coefficient of $E_{\varepsilon}$ is positive. We note that the function $t \rightarrow$ $2\left(\mu_{L}+1\right)\left(1+4 \sqrt{t} e^{C t}\right) N t$ is positive, strictly increasing for $t>0$ and vanish at 0 . Thus, if

$$
F_{0}=\left|L_{0}-y_{1}\right|<\rho
$$

then there exists $T_{l o c}>0$ such that the inequality

$$
2\left(\mu_{L}+1\right)\left(1+4 \sqrt{t} e^{C t}\right) N t+F_{0}<\rho
$$

takes place on $\left(0, T_{l o c}\right)$. Actually, $T_{l o c}$ is the solution to the equation

$$
2\left(\mu_{L}+1\right)\left(1+4 \sqrt{t} e^{C t}\right) N t+F_{0}=\rho .
$$

It remains to check if one can choose $y_{1}$ and $\rho$ to satisfy (4.10), taking into account the possible situations for $L_{0}$ and $L_{\min }$ which are fixed, with $L_{0} \leq L_{\min }$.

I. Let $L_{0} \leq \frac{L_{\min }}{2}$. We choose $L_{0}<y_{1}<\frac{L_{\min }}{2}$ and $\rho \in\left(y_{1}-L_{0}, y_{1}\right)$. Then, $\min \left\{y_{1}, L_{\min }-y_{1}\right\}=y_{1}$ and both (4.3) and (4.10) are satisfied.

II. Let $L_{0} \in\left(\frac{L_{\min }}{2}, L_{\min }\right)$. We choose $y_{1} \in\left(\frac{L_{\min }}{2}, L_{0}\right) \subset\left(\frac{L_{\min }}{2}, L_{\min }\right)$ and $\rho \in\left(L_{0}-y_{1}, L_{\min }-y_{1}\right)$. Then, $\min \left\{y_{1}, L_{\min }-y_{1}\right\}=L_{\min }-y_{1}$ and both (4.3) and (4.10) are satisfied.

Going back to (4.9) we can write that

$$
\begin{aligned}
& E_{\varepsilon}=\int_{0}^{T}\left|e_{\varepsilon}^{\prime}(t)\right| d t \leq \frac{\left(8\left(\mu_{L}+1\right) \sqrt{T} e^{C T}+4 \alpha_{L}\right) N^{2} T}{\rho-F_{0}-2\left(\mu_{L}+1\right)\left(1+4 \sqrt{T} e^{C T}\right) N T} \leq C, \\
& \text { for } T \in\left(0, T_{l o c}\right) \text {. }
\end{aligned}
$$

with $C$ independent of $\varepsilon$. This and (4.6) imply (4.1). Recalling (4.8) we obtain (4.2). This ends the proof. 
Remark 4.2 Now, we recall a few definitions and results necessary in the proof of the next theorem. We denote by $B V([0, T])$ the space of functions $v:[0, T] \rightarrow \mathbb{R}$ with bounded variation, that is

$$
\|v\|_{B V([0, T])}=\sup \left\{\sum_{i=0}^{M-1}\left|v\left(t_{i+1}\right)-v\left(t_{i}\right)\right| ; 0=t_{0}<t_{1}<\ldots<t_{M}=T\right\}<\infty
$$

and by $\mathcal{M}([0, T])$ the dual of the separable space $C([0, T])$. The space $\mathcal{M}([0, T])$ contains the bounded Radon measures defined on $[0, T]$. We also recall by the Lebesgue decomposition theorem that every $\mu \in\left(L^{\infty}(0, T)\right)^{*}$ can be uniquely written as

$$
\mu=\mu_{a}+\mu_{s},
$$

where $\mu_{a} \in L^{1}(0, T)$ and $\mu_{S}$ is a singular measure (see e.g. [10]). This means that for each $\varepsilon>0$ there exists a Lebesgue measurable set $\mathcal{S} \subset[0, T]$ with meas $([0, T] \backslash \mathcal{S}) \leq$ $\varepsilon$ and $\mu_{s}(\varphi)=0$ for all $\varphi \in L^{\infty}(\mathcal{S})$. The support (supp) of $\mu_{s}$ is the set of all $t \in[0, T]$ for which $\mu_{s}(\varphi) \neq 0$, for all $\varphi \in L^{\infty}(t-\delta, t+\delta)$, and all $\delta$ positive. Next, we recall that every $v \in B V([0, T])$ has a unique decomposition,

$$
v=v^{a}+v^{s}
$$

where $v^{a} \in A C[0, T]$ and $v^{s} \in B V([0, T])$. Here, $A C[0, T]$ is the space of absolutely continuous functions on $[0, T]$ and $v^{s}$ is a singular part (for instance it can be a jump function with bounded variation or a function with bounded variation with a.e. zero derivative).

We note that if $v \in B V([0, T])$, then its distributional derivative $\frac{d v}{d t}:=\mu$ belongs to $\left(L^{\infty}(0, T)\right)^{*}$, and in virtue of the Lebesgue decomposition, it is represented by the sum of the absolutely continuous part and the singular part

$$
\frac{d v}{d t}=\mu_{a}+\mu_{s}=\frac{d v^{a}}{d t}+\frac{d v^{s}}{d t} \in \mathcal{D}^{\prime}(0, T),
$$

where $\mathcal{D}^{\prime}(0, T)$ is the space of Schwartz distributions on $(0, T)$.

In the next theorem we shall pass to the limit in the approximating optimality conditions. To this end, we introduce the system

$$
\begin{aligned}
& p^{\prime}-k_{0}^{*} p+k_{0}^{*} q=0, \text { a.e. } t \in(0, T), \\
& q^{\prime}-\beta_{A} S^{*} p+k_{3}^{*} q+\sigma d+l_{A}^{*} e+\mu_{A} f=0, \text { a.e. } t \in(0, T), \\
& d^{\prime}-\beta_{I} S^{*} p+\beta_{I} S^{*} q-k_{2}^{*} d+l_{I}^{*} e+\mu_{I} f=0, \text { a.e. } t \in(0, T), \\
& e^{\prime}-\mu_{L} e+\mu_{L} f+v=-\alpha_{L}\left(L^{*}-L_{o b s}\right), \text { in } \mathcal{D}^{\prime}(0, T), \\
& f^{\prime}+\xi p-\xi f=-\alpha_{R}\left(R^{*}-R_{o b s}\right), \text { a.e. } t \in(0, T), \\
& p(T)=0, q(T)=0, d(T)=0, e(T)=0, f(T)=0,
\end{aligned}
$$


where

$$
\begin{aligned}
& v:=\alpha_{2} \lim _{\varepsilon \rightarrow 0}\left(\frac{1}{\varepsilon}\left(L_{\varepsilon}^{*}-L_{\text {test }}\right)^{+}\right) \text {weak* in }\left(L^{\infty}(0, T)\right)^{*}, \\
& v=v_{a}+v_{s}, v_{a} \in L^{1}(0, T), v_{s} \in \mathcal{M}([0, T]), \\
& k_{0}^{*}=\beta_{A} A^{*}+\beta_{I} I^{*}, k_{1}^{*}=\sigma+\mu_{A}+l_{A}^{*}, \\
& k_{2}^{*}=\mu_{I}+l_{I}^{*}, k_{3}^{*}=\beta_{A} S^{*}-k_{1}^{*} .
\end{aligned}
$$

We also define

$$
\widehat{K}(t)=\left\{y \in \mathbb{R} ;-\infty<y \leq L_{\text {test }}(t)\right\}, \widehat{\mathcal{K}}=\left\{v \in C([0, T]) ; v(t) \leq L_{\text {test }}(t), t \in[0, T]\right\} .
$$

We denote by $N_{\widehat{K}(t)}(z)$ the normal cone to $\widehat{K}(t)$ at $z$,

$$
N_{\widehat{K}(t)}(z)=\left\{\begin{array}{l}
0, \text { if } z<L_{\text {test }}(t) \\
\mathbb{R}^{+}, \text {if } z=L_{\text {test }}(t)
\end{array}\right.
$$

and by

$$
\mathcal{N}_{\widehat{\mathcal{K}}}(\zeta)=\{\eta \in \mathcal{M}([0, T]) ; \eta(\zeta-z) \geq 0, \forall z \in \widehat{\mathcal{K}}\}
$$

the normal cone to $\widehat{\mathcal{K}}$ at $z$, where $\eta(\zeta-z)$ is the value of the measure $\eta \in \mathcal{M}([0, T])$ at $(\zeta-z) \in C([0, T])$.

Theorem 4.3 below is the main result.

Theorem 4.3 Let $\left(l_{A}^{*}, l_{I}^{*}\right)$ be optimal in $(P)$ with the corresponding state $X^{*}$. Then, if $T<T_{\text {loc }}$ defined in (4.12), the optimality conditions for problem $(P)$ read:

$$
l_{A}^{*}(t)=P_{[0,1]}\left(\frac{1}{\alpha_{1}} A^{*}(q-e)(t)\right), l_{I}^{*}(t)=P_{[0,1]}\left(\frac{1}{\alpha_{1}} I^{*}(d-e)(t)\right) \text {, a.e. } t \in(0, T),
$$

where $(p, q, d, e, f)$ is the solution of the dual system (4.17)-(4.24) with

$$
(p, q, d, f) \in\left(W^{1, \infty}(0, T)\right)^{4}, e \in B V\left([0, T], e^{\prime} \in \mathcal{M}([0, T]),\right.
$$

where $e=e^{a}+e^{s}, e^{a} \in A C([0, T]), e^{s} \in B V([0, T]), e^{\prime}=\left(e^{\prime}\right)_{a}+\left(e^{\prime}\right)_{s},\left(e^{\prime}\right)_{a} \in$ $L^{1}(0, T),\left(e^{\prime}\right)_{s} \in \mathcal{M}([0, T])$,

$$
\begin{aligned}
& \left(e^{\prime}\right)_{a}(t)+v_{a}(t)=\left(\mu_{L} e-\mu_{L} f\right)(t), \text { a.e. } t \in(0, T), \\
& \left(e_{s}\right)^{\prime}+v_{s}=0, \quad \text { in } \mathcal{D}^{\prime}(0, T),
\end{aligned}
$$

where

$$
v_{a}(t) \in N_{\widehat{K}(t)}\left(L^{*}(t)\right) \text {, a.e. } t \in(0, T),
$$




$$
v_{s}(\varphi) \geq 0 \text { and supp } v_{s} \subset\left\{t \in[0, T] ; L^{*}(t)=L_{\text {test }}(t)\right\}
$$

Proof We shall establish some estimates in order to pass to the limit in the approximating optimality conditions determined in Proposition 3.3. Let $T<T_{l o c}$, let $\left(l_{A}^{*}, l_{I}^{*}\right)$ be optimal in $(P)$ with the corresponding state $X^{*}$ and let us consider $\left\{\left(l_{A, \varepsilon}^{*}, l_{I, \varepsilon}^{*}\right), X_{\varepsilon}^{*}\right\}$ optimal in $\left(P_{\varepsilon}\right)$. Recalling Proposition 3.2 and the continuity property from Proposition 2.1, we have $l_{A, \varepsilon}^{*} \rightarrow l_{A}^{*}, l_{I, \varepsilon}^{*} \rightarrow l_{I}^{*}$ weak* in $L^{\infty}(0, T)$, strongly in $L^{2}(0, T)$ and

$$
X_{\varepsilon}^{*} \rightarrow X^{*} \text { weak* in }\left(W^{1, \infty}(0, T)\right)^{5} \text { and uniformly on }[0, T] \text {. }
$$

By (4.1) and (4.2) each component of the solution to the dual system follows to be bounded in $L^{\infty}(0, T)$. Moreover, by (3.8)-(3.10), (3.12) it follows that $\left(p_{\varepsilon}^{\prime}\right)_{\varepsilon},\left(q_{\varepsilon}^{\prime}\right)_{\varepsilon},\left(d_{\varepsilon}^{\prime}\right)_{\varepsilon},\left(f_{\varepsilon}^{\prime}\right)_{\varepsilon}$ are bounded in $L^{\infty}(0, T)$, so that, on a subsequence,

$$
\begin{aligned}
p_{\varepsilon} \rightarrow & p, q_{\varepsilon} \rightarrow q, d_{\varepsilon} \rightarrow d, f_{\varepsilon} \rightarrow f \text { weak* }^{*} \text { in } W^{1, \infty}(0, T) \\
& \text { and uniformly in }[0, T], \text { as } \varepsilon \rightarrow 0 .
\end{aligned}
$$

The component $\left(e_{\varepsilon}\right)_{\varepsilon}$ is bounded in $L^{\infty}(0, T)$ by (4.6) and its derivative is bounded in $L^{1}(0, T)$, by (4.1). These imply that $e_{\varepsilon} \in B V([0, T])$ and by Helly's theorem (see e.g., [11], p. 47) it follows that there is $e \in B V([0, T])$ such that

$$
e_{\varepsilon}(t) \rightarrow e(t), \text { for all } t \in[0, T], \text { as } \varepsilon \rightarrow 0 \text {. }
$$

Going back to (3.11) we deduce that $\frac{1}{\varepsilon}\left(L_{\varepsilon}^{*}-L_{\text {test }}\right)^{+} \in L^{1}(0, T)$. Now, we assert that $\left(e_{\varepsilon}^{\prime}\right)_{\varepsilon}$ and $\left(\frac{1}{\varepsilon}\left(L_{\varepsilon}^{*}-L_{\text {test }}\right)^{+}\right)_{\varepsilon}$ are weak* compact in $\left(L^{\infty}(0, T)\right)^{*}$, the dual of $L^{\infty}(0, T)$. This is pointed out in the proof of Corollary 2B in [10], but this assertion does not follow directly from Alaoglu theorem. An argument can be found in [12], and we resume it below.

Let us consider the linear operator $\Psi: C([0, T]) \rightarrow L^{\infty}(0, T), \Psi z=\widetilde{\Psi}$, which maps a continuous function into the corresponding class of equivalence $\widetilde{\Psi}$ (of all functions a.e. equal). Its adjoint $\Psi^{*}:\left(L^{\infty}(0, T)\right)^{*} \rightarrow \mathcal{M}([0, T])$, is defined by $\left(\Psi^{*} \mu\right)(z):=\mu\left(\Psi_{z}\right)$ for any $z \in C([0, T])$. If $\left(\mu_{n}\right)_{n}$ is bounded in $\left(L^{\infty}(0, T)\right)^{*}$ and also in $\mathcal{M}([0, T])$, then $\left(\Psi^{*} \mu_{n}\right)_{n}$ is bounded in $\mathcal{M}([0, T])$ and using the Alaoglu theorem it follows that $\left(\Psi^{*} \mu_{n}\right)_{n}$ is weak* sequentially compact in $\mathcal{M}([0, T])$. Therefore, it follows that $\left(\mu_{n}\right)_{n}$ is weak* sequentially compact in $\mathcal{M}([0, T])$. Passing to the limit in $\mu_{n}(\Psi z)=\left(\Psi^{*} \mu_{n}\right)(z)$ we get $\mu(\Psi z)=\left(\Psi^{*} \mu\right)(z)$ for any $\widetilde{\Psi} \in L^{\infty}(0, T)$ which is of the form $\Psi z$ with $z \in C([0, T])$. Then, due to the Hahn-Banach theorem, $\mu$ can be extended to all $L^{\infty}(0, T)$ and so we conclude that $\left(\mu_{n}\right)_{n}$ is weak* sequentially compact in $\left(L^{\infty}(0, T)\right)^{*}$.

Therefore, one can extract a subsequence such that

$$
e_{\varepsilon}^{\prime} \rightarrow e^{\prime}, \frac{\alpha_{2}}{\varepsilon}\left(L_{\varepsilon}^{*}-L_{\text {test }}\right)^{+} \rightarrow v \text { weak }^{*} \text { in }\left(L^{\infty}(0, T)\right)^{*} \subset \mathcal{M}([0, T]) .
$$


Thus, relying on (4.33)-(4.35), we can pass to the limit in (3.8)-(3.13) and obtain (4.17)-(4.23).

Now, we move to (3.14), or more exactly in (3.18) and (3.19) and pass to the limit. The left-hand side of (3.18) converges and since the normal cone is maximal monotone, hence strongly-strongly closed it follows that

$$
\left(-I^{*}(e-d)-\alpha_{1} l_{I}^{*}\right)(t) \in N_{[0,1]}\left(l_{I}^{*}(t)\right)
$$

which implies the second relation in (4.27). Similarly, we proceed in (3.19) and obtain

$$
\left(-A^{*}(e-q)-\alpha_{1} l_{A}^{*}\right)(t) \in N_{[0,1]}\left(l_{A}^{*}(t)\right)
$$

whence we get the first relation in (4.27).

Finally, we want to interpret Eq. (4.20). Since $z^{+}$is the subdifferential of the function $\frac{1}{2}\left(z^{+}\right)^{2}$, we can write

$$
\begin{aligned}
& \frac{\alpha_{2}}{\varepsilon} \int_{0}^{T}\left(L_{\varepsilon}^{*}-L_{\text {test }}\right)^{+}\left(\left(L_{\varepsilon}^{*}-L_{\text {test }}\right)-\left(z(t)-L_{\text {test }}\right)\right) d t \\
& \geq \frac{\alpha_{2}}{2 \varepsilon} \int_{0}^{T}\left(\left(\left(L_{\varepsilon}^{*}-L_{\text {test }}\right)^{+}\right)^{2}-\frac{1}{2}\left(\left(z-L_{\text {test }}\right)^{+}\right)^{2}\right) d t \geq 0,
\end{aligned}
$$

for all $z \in \widehat{\mathcal{K}}$. At limit we obtain

$$
v\left(L^{*}-z\right) \geq 0, \text { for all } z \in \widehat{\mathcal{K}},
$$

whence, $v \in \mathcal{N}_{\widehat{\mathcal{K}}}\left(L^{*}\right)$.

Since $v \in\left(L^{\infty}(0, T)\right)^{*} \subset \mathcal{M}([0, T])$, recalling (4.14) and (4.16), we can represent $v=v_{a}+v_{s}$, where $v_{a}$ is the absolutely continuous part (in the sense of measure) and $v_{s}$ is the singular part of $v$. Also, $e^{\prime} \in \mathcal{M}([0, T])$ and $e^{\prime}=\left(e^{\prime}\right)_{a}+\left(e^{\prime}\right)_{s}$ where $\left(e^{\prime}\right)_{a}=e_{a}^{\prime} \in L^{1}(0, T)$. Then, (4.20) can be rewritten as in (4.29)-(4.30). Relation (4.36) implies that $v_{a}(t) \in N_{\widehat{K}(t)}\left(L^{*}(t)\right)$, a.e. $t \in(0, T)$, that is

$$
v_{a}(t)=0 \text { if } L^{*}(t)<L_{\text {test }}(t), v_{a}(t) \geq 0 \text { if } L^{*}(t)=L_{\text {test }}(t)
$$

As regards $v_{s}$ we have

$$
v_{s}(\varphi) \geq 0, v_{s}(\varphi)=0 \text { if } \varphi \in \stackrel{\circ}{\mathcal{K}}
$$

where $\stackrel{\circ}{\widehat{\mathcal{K}}}=\left\{\varphi \in C([0, T]) ; \varphi(t)<L_{\text {test }}(t)\right.$ for $\left.t \in[0, T]\right\}$ is the interior of $\widehat{\mathcal{K}}$.

Here is the argument. Relation (4.36) can be still written

$$
\int_{0}^{T} v_{a}(t)\left(L^{*}(t)-z(t)\right) d t+v_{s}\left(L^{*}-z\right) \geq 0 \text { for all } z \in \widehat{\mathcal{K}} .
$$


Since $v_{s}$ is singular with respect to the Lebesgue measure, this inequality implies separately that each term in (4.39) is greater or equal to 0 .

If $t_{0} \in(0, T)$ is such that $L^{*}\left(t_{0}\right) \leq L_{\text {test }}\left(t_{0}\right)$, then choosing in the first part

$$
z(t)= \begin{cases}z_{0}, & \text { for } t \in\left(t_{0}-\varepsilon, t_{0}+\varepsilon\right) \\ L^{*}(t), & \text { otherwise, }\end{cases}
$$

where $z_{0} \leq L_{\text {test }}\left(t_{0}\right)$, and $\varepsilon>0$ is arbitrary small, we get

$$
\frac{1}{\varepsilon} \int_{t_{0}-\varepsilon}^{t_{0}+\varepsilon} v_{a}(t)\left(L^{*}(t)-z_{0}\right) d t \geq 0, \text { for all } \varepsilon>0
$$

Letting $\varepsilon \rightarrow 0$, we obtain $v_{a}\left(t_{0}\right)\left(L^{*}\left(t_{0}\right)-z_{0}\right) \geq 0$ for almost all Lebesgue points $t_{0}$ and since $z_{0}$ is arbitrary, the relation $v_{a}(t) \in N_{\widehat{K}(t)}\left(L^{*}(t)\right)$, a.e. $t \in(0, T)$ is inferred.

Now, again by (4.39) it follows $v_{s}\left(L^{*}-z\right) \geq 0$ and so (4.38) is implied.

The measure $v_{s}$ has the support on the boundary of $\widehat{\mathcal{K}}$. Recalling that

$$
\operatorname{supp}_{s}=\left\{\Sigma \subset[0, T] ; v_{s} \neq 0 \text { on } \Sigma\right\}
$$

it follows that supp $v_{s} \subset\left\{t \in(0, T) ; z(t)=L_{\text {test }}(t)\right\}$. Thus, we actually get (4.32), as claimed.

Remark 4.4 In Theorem 4.3, $e^{a}$ and $e^{s}$ are the absolutely continuous part and singular part, respectively, of the function $e \in B V([0, T])$, while $\left(e^{\prime}\right)_{a}=\left(e^{a}\right)^{\prime} \in L^{1}(0, T)$ and $\left(e^{\prime}\right)_{s}=\left(e^{s}\right)^{\prime}$ are the derivative in the sense of distributions on $(0, T)$. By (4.27) we see that the optimally identified inputs $l_{A}^{*}$ and $l_{I}^{*}$ are with bounded variation on $[0, T]$ and their derivatives $\left(l_{A}^{*}\right)^{\prime}$ and $\left(l_{I}^{*}\right)^{\prime}$ are singular on the saturation set $\left\{t ; L^{*}(t)=L_{\text {test }}(t)\right\}$. This phenomenon is typical for state constraints optimal control problems (see [8]). Therefore, we can interpret that the optimal rates $l_{A}^{*}$ and $l_{I}^{*}$ are absolutely continuous on the set $\left\{t ; L^{*}(t)<L_{\text {test }}(t)\right\}$ where the number of positive detected and isolated individuals is less than the number of tests, and become singular, having possible jumps at the moments when all tested individuals are found infected and are isolated.

\section{Conclusions}

We solved an inverse problem for the identification of the detection-isolation rates of an epidemic model, on the basis of the observations made for the confirmed isolated and recovered compartments. Using these determined rates one can estimate the number of the individuals in all compartments, especially in the infectious classes. This can offer information about the epidemiologic situation, which may help the policies for modifying these rates in view of a better disease containment. In this respect, an additional estimation of the rates $l_{A}$ and $l_{I}$ that may contribute to the minimization 
of the total infectious population within a time interval $[0, T]$, could be done via the optimization problem

$\operatorname{minimize}\left\{J_{1}\left(l_{A}, l_{I}\right) ; l_{A}(t) \in[0,1], l_{I}(t) \in[0,1]\right.$ a.a. $\left.t \in(0, T), 0 \leq L(t) \leq L_{\text {test }}(t)\right\}$

subject to (1.1)-(1.7), where

$$
J_{1}\left(l_{A}, l_{I}\right)=\alpha_{0} \int_{0}^{T}(A(t)+I(t)) d t+\frac{1}{2} \int_{0}^{t}\left(l_{A}^{2}(t)+l_{I}^{2}(t)\right) d t .
$$

This problem can be similarly treated as problem $(P)$ with slight differences in some calculations due to the new cost functional. The appropriate approximating problem is

Minimize $\left\{J_{1, \varepsilon}\left(l_{A}, l_{I}\right) ; l_{A} \in[0,1], l_{I} \in[0,1]\right.$ a.e. on $\left.(0, T)\right\}$

subject to (1.1)-(1.7), where

$$
\begin{aligned}
J_{1, \varepsilon}\left(l_{A}, l_{I}\right)= & \alpha_{0} \int_{0}^{T}(A(t)+I(t)) d t+\frac{\alpha_{1}}{2} \int_{0}^{T}\left(l_{A}^{2}(t)+l_{I}^{2}(t)\right) d t \\
& +\frac{1}{2} \int_{0}^{T}\left\{\left(l_{A}(t)-l_{A}^{*}(t)\right)^{2}+\left(l_{I}(t)-l_{I}^{*}(t)\right)^{2}\right\} d t \\
& +\frac{\alpha_{2}}{2 \varepsilon} \int_{0}^{T}\left(\left(L(t)-L_{\text {test }}(t)\right)^{+}\right)^{2} d t .
\end{aligned}
$$

The approximating optimality conditions are calculated as in Proposition 3.3, the approximating optimal controls are still given by (3.14), but the dual system is

$$
\begin{aligned}
& p_{\varepsilon}^{\prime}-k_{0, \varepsilon} p_{\varepsilon}+k_{0, \varepsilon} q_{\varepsilon}=0, \\
& q_{\varepsilon}^{\prime}-\beta_{A} S_{\varepsilon}^{*} p_{\varepsilon}+k_{3, \varepsilon} q_{\varepsilon}+\sigma d_{\varepsilon}+l_{A, \varepsilon}^{*} e_{\varepsilon}+\mu_{A} f_{\varepsilon}=-\alpha_{0}, \\
& d_{\varepsilon}^{\prime}-\beta_{I} S_{\varepsilon}^{*} p_{\varepsilon}+\beta_{I} S_{\varepsilon}^{*} q_{\varepsilon}-k_{2, \varepsilon} d_{\varepsilon}+l_{I, \varepsilon}^{*} e_{\varepsilon}+\mu_{I} f_{\varepsilon}=-\alpha_{0}, \\
& e_{\varepsilon}^{\prime}-\mu_{L} e_{\varepsilon}+\mu_{L} f_{\varepsilon}+\frac{\alpha_{2}}{\varepsilon}\left(L_{\varepsilon}^{*}-L_{\text {test }}\right)^{+}=0, \\
& f_{\varepsilon}^{\prime}+\xi p_{\varepsilon}-\xi f_{\varepsilon}=0,
\end{aligned}
$$

for a.a. $t>0$, with the final conditions

$$
p_{\varepsilon}(T)=0, q_{\varepsilon}(T)=0, d_{\varepsilon}(T)=0, e_{\varepsilon}(T)=0, f_{\varepsilon}(T)=0 .
$$

Theorem 4.3 remains true, and the dual system (4.17)-(4.22) has the only modification regarding the free terms which consist now in the vector $\left(0,-\alpha_{0},-\alpha_{0}, 0,0\right)$. 


\section{References}

1. Cascella, M., Rajnik, M., Cuomo, A., Dulebohn, S.C., Di Napoli, R.: Features, Evaluation and Treatment of Coronavirus (COVID-19). StatPearls Publishing, Napoli (2020)

2. Armstrong, E., Runge, M., Gerardin, J.: Identifying the measurements required to estimate rates of COVID-19 transmission, infection, and detection, using variational data assimilation. Infectious Dis. Modell. 6, 133-147 (2021)

3. Giordano, G., Blanchini, F., Bruno, R., Colaneri, P., Di Filippo, A., Di Matteo, A., Colaneri, M.: Modelling the COVID-19 epidemic and implementation of population-wide interventions in Italy. Letters 26(6), 855-860 (2020). https://doi.org/10.1038/s41591-020-0883-7

4. Yang, C., Wang, J.: Modeling the transmission of COVID-19 in the US: a case study. Infectious Dis. Model. 6, 195-211 (2021)

5. Linton, N., Kobayashi, T., Yang, Y., Hayashi, K., Akhmetzhanov, A.R., Jung, S.M., Yuan, B., Kinoshita, R., Nishiura, H.: Incubation period and other epidemiological characteristics of 2019 novel coronavirus infections with right truncation: a statistical analysis of publicly available case data. J. Clin. Med. 9, 538 (2020)

6. Fuhrmann, J., Barbarossa, M.V.: The significance of case detection ratios for predictions on the outcome of an epidemic: a message from mathematical modelers. Archiv. Public Health 1(6), 78 (2020)

7. Peto, J.: Covid-19 mass testing facilities could end the epidemic rapidly. Br. Med. J. 368, m1163 (2020)

8. Rockafeller, R.T.: State constraints in convex control problems of Bolza. SIAM J. Control 10, 691-715 (1972)

9. Barbu, V.: Differential Equations. Springer, London (2016)

10. Rockafeller, R.T.: Integrals which are convex functionals, II. Pac. J. Math. 39, 439-469 (1971)

11. Barbu, V., Precupanu, T.: Convexity and Optimization in Banach Spaces, 4 th edn. Springer, New York (2012)

12. Barbu, V., Da Prato, G., Tubaro, L.: The stochastic reflection problem in Hilbert spaces. Commun. Partial Differ. Equ. 37, 352-367 (2012)

Publisher's Note Springer Nature remains neutral with regard to jurisdictional claims in published maps and institutional affiliations. 